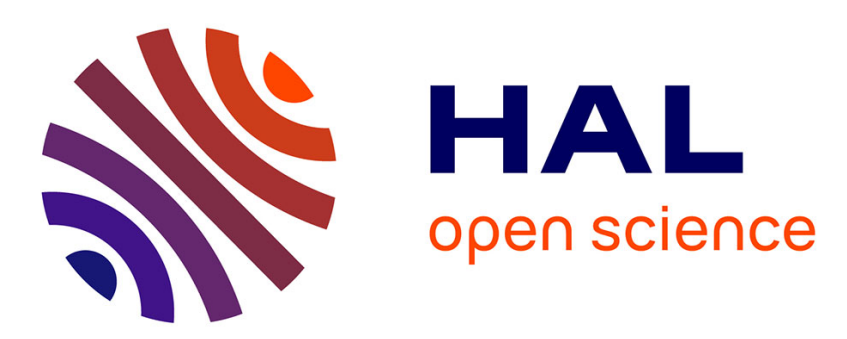

\title{
Asymptotics of the eigenvalues of the Dirichlet-Laplace problem in a domain with thin tube excluded \\ Xavier Claeys
}

\section{To cite this version:}

Xavier Claeys. Asymptotics of the eigenvalues of the Dirichlet-Laplace problem in a domain with thin tube excluded. Quarterly of Applied Mathematics, 2016, 74 (4), pp.595-605. hal-01120422v2

\section{HAL Id: hal-01120422 \\ https://hal.science/hal-01120422v2}

Submitted on 7 Apr 2016

HAL is a multi-disciplinary open access archive for the deposit and dissemination of scientific research documents, whether they are published or not. The documents may come from teaching and research institutions in France or abroad, or from public or private research centers.
L'archive ouverte pluridisciplinaire HAL, est destinée au dépôt et à la diffusion de documents scientifiques de niveau recherche, publiés ou non, émanant des établissements d'enseignement et de recherche français ou étrangers, des laboratoires publics ou privés. 


\title{
Asymptotics of the eigenvalues of the Dirichlet- Laplace problem in a domain with thin tube excluded
}

\author{
X.Claeys ${ }^{* \dagger}$
}

\begin{abstract}
We consider a Laplace problem with Dirichlet boundary condition in a three dimensional domain containing an inclusion taking the form of a thin tube with small thickness $\delta$. We prove convergence in operator norm of the resolvent of this problem as $\delta \rightarrow 0$, establishing that the perturbation induced by the inclusion on the resolvent is not greater than $O\left(|\ln \delta|^{-\gamma}\right)$ for some $\gamma>0$. We deduce convergence of the eigenvalues of the perturbed operator toward the limit operator.
\end{abstract}

\section{Introduction}

Analysis of singular perturbations of elliptic boundary value problems by inclusions of small volume has received a lot of attention in the past decades due to its numerous applications to modeling and numerical simulation of multi-scale problems, and its possible use in efficient inverse problem [1,2] or shape optimization [20] strategies.

For Laplace equation, many works of the existing literature have been dedicated to asymptotic analysis involving the presence of an inclusion that has small size in all directions of space (typically small balls), in the case of either impenetrable (homogeneous Dirichlet, Neumann, or Robin) or penetrable (transmission problem) boundary conditions. Such asymptotics have been provided for both 2-D and 3-D problems, in the case of either a single inclusion or many, see $[19,18,17,16,10]$. The books $[14,9]$ are landmarks on this type of problem.

As regards Laplace equation perturbed by a small inclusion that takes the form of a thin tube, much less work is available in the literature. Some works can be found in the case of a tube centered around a smooth closed contour without self-intersecting point. The analysis of Laplace equation in 3-D with Neumann and Dirichlet boundary condition was provided in $[5,6]$ where the author considers either a tube centered at a non-self-intersecting smooth closed contour, or a tube centered at a straight segment. This analysis is refined and generalized to arbitrary dimensions in [15] and [14, Chap.12]. Similar results were established for acoustics and Helmholtz equation in [3, 7, 22].

It should be pointed out that, in the case of a 3-D Laplace equation perturbed by a thin elongated inclusion, the cases of the homogeneous Dirichlet or Robin boundary condition lead to much more challenging analysis than the case of Neumann or transmission conditions: while Neumann/transmission conditions lead to rather standard asymptotic constructions, matching of asymptotics in the Dirichlet/Robin case leads to an ill-posed 1-D integral equation.

${ }^{*}$ Laboratoire Jacques-Louis Lions, UPMC Univ. Paris 6 and CNRS UMR 7598, 75005, Paris, France

${ }^{\dagger}$ INRIA-Paris-Rocquencourt, EPC Alpines, Le Chesnay Cedex, France 
On the other hand, asymptotic analysis of the Laplace problem with Dirichlet boundary condition has many important applications in particular in electrical engineering and antenna based electric/electronic devices, as it is commonly taken as a rough model for electromagnetic diffraction by perfectly conducting thin wires, see for example [11, Chap.6]. In this context, the study of eigenvalues is of particular importance as it is related to resonance phenomena. Yet, in the case of thin elongated inclusions with Dirichlet boundary condition, only Planida [21] has addressed this issue so far. In this reference, the author establishes convergence of eigenvalues in the case where the inclusion is centered around a smooth closed curve that does not self-intersect. However no explicit rate of convergence is provided.

In the present article, we study Laplace equation with homogeneous Dirichlet condition, and the associated eigenvalue problem, perturbed by the presence of a small elongated inclusion that takes the form of a thin tube. We establish convergence in the operator norm, of the resolvent of the perturbed problem toward the resolvent of the limit problem. A first novel ingredient here compared to previously established results concern the geometrical configurations under consideration that only require the inclusion to concentrate around a parametrized curve. Contrary to pre-existing works, the present analysis does not require the inclusion to be connected, or admit any symmetry of revolution. The limit curve considered here may admit self-crossing points which, to our knowledge, has never been investigated in the existing literature (at least in the case of an homogeneous Dirichlet boundary condition). In addition, we establish a convergence result (with error estimates) for eigenvalues with explicit error estimate showing that the presence of an elongated inclusion induces a shift of order $O\left(|\ln \delta|^{-\gamma}\right)$ of the eigenvalues, for some $\gamma>0$.

The outline of the present article is as follows. In Section 2 we describe in detail the geometry under consideration. In Section 3 we introduce weighted $\delta$-dependent norms adapted to our analysis, and establish a stability property for the Laplace operator in terms of these norms. The next section is dedicated to proving Hardy type inequalities that will be necessary for the subsequent error estimates. In the last section we derive an upper bound for the difference, in operator norm, between the resolvents of the perturbed problem and the limit problem.

\section{Geometry under consideration}

We start by describing in detail the geometrical setting under consideration. Let $\Omega \subset \mathbb{R}^{3}$ refer to a Lipschitz domain. Consider a $\mathscr{C}^{1}$-function $\gamma: \mathbb{R} \mapsto \Omega$ that is $L$-periodic for some $L>0$, and such that $0<\alpha_{-} \leq\left|\frac{d \gamma}{d z}(z)\right| \leq \alpha_{+}, \forall z \in \mathbb{R}$ for fixed constants $\alpha_{ \pm}>0$. Then we set $\Gamma:=\gamma(\mathbb{R})$, which is a Lipschitz curve. We shall need to refer to the distance function

$$
d(\boldsymbol{x}):=\inf _{\boldsymbol{y} \in \Gamma}|\boldsymbol{x}-\boldsymbol{y}| .
$$

Then we consider $\left(\Xi_{\delta}\right)_{\delta>0}$ as any family of Lipschitz domains such that there exists a fixed constant $C>0$ independent of $\delta$ for which

$$
\sup _{\boldsymbol{x} \in \bar{\Xi}_{\delta}} d(\boldsymbol{x}, \Gamma) \leq C \delta .
$$

This condition imposes that, as $\delta \rightarrow 0$, the sets $\Xi_{\delta}$ are more and more concentrated around $\Gamma$. Changing the index parameter $\delta$ through multiplication by some factor if necessary, we may assume that $C<1$ in (2) without restricting generality. Then we denote

$$
\Omega_{\delta}:=\Omega \backslash \bar{\Xi}_{\delta} .
$$


The geometrical configurations described above may be used to consider resonance problems in cavities containing inclusions whose geometry is similar to wires. Note that we do not require $\gamma$ to be one-to-one over a period so that, under the assumptions above, the curve $\Gamma$ may self-intersect. Note also that $\Xi_{\delta}$ do not need to be connected and may degenerate, as $\delta \rightarrow 0$, to only a part of $\Gamma$ with tips. As a consequence, the present setting covers such geometrical configurations as the ones presented in pictures below.
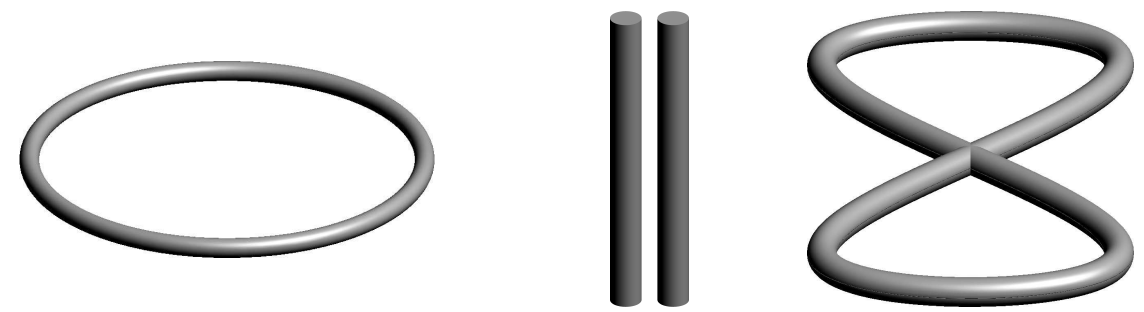

Figure 1 Three examples of geometrical configurations covered by the analysis presented here.

\section{Weighted Sobolev spaces}

Given any bounded open Lipschitz domain $\omega \subset \mathbb{R}^{3}$, the set $\mathrm{L}^{2}(\omega)$ shall refer to the space of square integrable functions over $\omega$ equipped with the pairing $u, v \mapsto(u, v)_{\omega}:=\int_{\omega} u v d \boldsymbol{x}$ and the norm $\|u\|_{\mathrm{L}^{2}(\omega)}=\sqrt{(u, \bar{u})_{\omega}}$. As usual, $\mathrm{H}_{0}^{1}(\omega)$ shall refer to the completion of $\mathscr{C}_{0}^{\infty}(\omega):=\left\{\varphi \in \mathscr{C}^{\infty}\left(\mathbb{R}^{3}\right), \varphi=0\right.$ on $\mathbb{R}^{3} \backslash \bar{\omega}, \operatorname{supp}(\varphi)$ bounded $\}$ for the norm $\|\varphi\|_{\mathrm{H}_{0}^{1}(\omega)}:=$ $\|\varphi\|_{\mathrm{L}^{2}(\omega)}+\|\nabla \varphi\|_{\mathrm{L}^{2}(\omega)}$. Finally $\mathrm{H}^{-1}(\omega)$ will refer to the topological dual to $\mathrm{H}_{0}^{1}(\omega)$.

For a given parameter $\delta>0$ small enough, we are interested in studying the eigenvalues of the operator $\mathrm{A}_{\delta}: \mathrm{H}_{0}^{1}\left(\Omega_{\delta}\right) \rightarrow \mathrm{H}^{-1}\left(\Omega_{\delta}\right)$ defined by

$$
\left\langle\mathrm{A}_{\delta}(u), v\right\rangle:=(\nabla u, \nabla v)_{\Omega} \quad \forall u, v \in \mathrm{H}_{0}^{1}\left(\Omega_{\delta}\right) .
$$

We shall compare its eigenvalues with those of the limit operator $\mathrm{A}_{0}: \mathrm{H}_{0}^{1}(\Omega) \rightarrow \mathrm{H}^{-1}(\Omega)$ that is defined similarly but in a domain without any perturbation i.e. $\left\langle\mathrm{A}_{0}(u), v\right\rangle:=$ $(\nabla u, \nabla v)_{\Omega} \forall u, v \in \mathrm{H}_{0}^{1}(\Omega)$.

To study these two operators, we need to introduce adapted $\delta$-dependent weighted Sobolev norms. These are not classical weighted norms such as those characterizing Kondratiev's spaces, see e.g. [13]. The norms we consider here involve $\delta$-dependent logarithmic weights: given a $\beta \in \mathbb{R}$, and a Lipschitz open set $\omega \subset \mathbb{R}^{2}$, we define

$$
\begin{aligned}
&\|v\|_{\mathrm{V}_{\beta, \delta}^{1}(\omega)}^{2}:=|v|_{\mathrm{V}_{\beta, \delta}^{1}(\omega)}^{2}+|v|_{\mathrm{V}_{\beta, \delta}^{0}(\omega)}^{2} \\
& \text { where } \quad|v|_{\mathrm{V}_{\beta}^{0}(\omega)}^{2}:=\int_{\omega}\left|\ln d_{\delta}(\boldsymbol{x})\right|^{-2 \beta} \frac{|v(\boldsymbol{x})|^{2}}{\left|d_{\delta}(\boldsymbol{x}) \ln d_{\delta}(\boldsymbol{x})\right|^{2}} d \boldsymbol{x} \\
& \qquad\left.v\right|_{\mathrm{V}_{\beta}^{1}(\omega)} ^{2}:=\int_{\omega}\left|\ln d_{\delta}(\boldsymbol{x})\right|^{-2 \beta}|\nabla v|^{2} d \boldsymbol{x} \\
& \text { with } \quad d_{\delta}(\boldsymbol{x}):=d_{\star}(\boldsymbol{x})+\delta \quad \text { where } \quad d_{\star}(\boldsymbol{x}):=\min \left(d(\boldsymbol{x}), e^{-1 / 2) .}\right.
\end{aligned}
$$

For $\delta>0, \beta \in \mathbb{R}$ fixed, the norm above is obviously equivalent to \|\|$_{\mathrm{H}^{1}(\omega)}$. However these norms "behave" differently as $\delta \rightarrow 0$. The norms (4) are specifically tailored for our analysis. 
The condition $d_{\star}(\boldsymbol{x}) \leq e^{-1} / 2 \forall \boldsymbol{x} \in \Omega$ guarantees that $\left|d_{\star}(\boldsymbol{x}) \ln d_{\star}(\boldsymbol{x})\right| \leq\left|d_{\delta}(\boldsymbol{x}) \ln d_{\delta}(\boldsymbol{x})\right|$ for all $\boldsymbol{x} \in \Omega$ and for $\delta<e^{-1} / 2$. On the other hand, recall that $d$ is a distance function to a compact set so it is Lipschitz with $\|\nabla d\|_{L^{\infty}(\Omega)}=1$, see [4, Prop.1D.4] for example. Hence $d_{\star}$ is a Lipschitz function, and $d_{\delta}$ is non-vanishing with $d_{\delta}(\boldsymbol{x}) \geq \delta, \forall \boldsymbol{x} \in \Omega$. We deduce that $\Upsilon_{\beta}^{\delta}(v):=v(\boldsymbol{x}) \ln ^{\beta} d_{\delta}(\boldsymbol{x})$ isomorphically maps $\mathrm{H}_{0}^{1}(\Omega)$ onto itself, and there exist constants $c_{ \pm}, \delta_{0}>0$ independent of $\delta$ such that

$$
0<c_{-} \leq \frac{\left\|\Upsilon_{\beta}^{\delta}(v)\right\|_{\mathrm{V}_{\beta, \delta}^{1}(\Omega)}}{\|v\|_{\mathrm{V}_{0, \delta}^{1}(\Omega)}} \leq c_{+} \quad \forall v \in \mathrm{H}_{0}^{1}(\Omega), \quad \forall \delta \in\left(0, \delta_{0}\right) .
$$

We shall also consider the corresponding dual norms, each of which providing a norm for $\mathrm{H}^{-1}(\Omega)$, and carrying at the same time some dependency with respect to $\delta$,

$$
\|f\|_{\mathrm{V}_{\beta, \delta}^{-1}(\omega)}:=\sup _{v \in \mathrm{H}_{0}^{1}(\omega) \backslash\{0\}} \frac{|\langle f, v\rangle|}{\|v\|_{\mathrm{V}_{-\beta, \delta}^{1}(\omega)}} .
$$

Let us examine the continuity properties of the operators $A_{0}, A_{\delta}$ with respect to these norms. First of all, both $\mathrm{A}_{0}$ and $\mathrm{A}_{\delta}$ easily appear as uniformly continuous with respect to these norms i.e. a direct calculus shows that

$$
\limsup _{\delta \rightarrow 0}\left(\sup _{v \in \mathrm{H}_{0}^{1}(\Omega) \backslash\{0\}} \frac{\left\|\mathrm{A}_{0}(v)\right\|_{\mathrm{V}_{\beta, \delta}^{-1}(\Omega)}}{\|v\|_{\mathrm{V}_{\beta, \delta}^{1}(\Omega)}}+\sup _{v \in \mathrm{H}_{0}^{1}\left(\Omega_{\delta}\right) \backslash\{0\}} \frac{\left\|\mathrm{A}_{\delta}(v)\right\|_{\mathrm{V}_{\beta, \delta}^{-1}\left(\Omega_{\delta}\right)}}{\|v\|_{\mathrm{V}_{\beta, \delta}^{1}\left(\Omega_{\delta}\right)}}\right)<+\infty .
$$

Uniform invertibility of these operators also hold under certain hypothesis on the weight exponent $\beta$.

\section{Proposition 3.1.}

There exists $\beta_{\star}>0$ such that, for $|\beta|<\beta_{\star}$, the inverses of both $\mathrm{A}_{0}$ and $\mathrm{A}_{\delta}$ remain uniformly bounded with respect to $\delta$ in terms of the weighted norms

$$
\limsup _{\delta \rightarrow 0}\left(\sup _{f \in \mathrm{H}^{-1}(\Omega) \backslash\{0\}} \frac{\left\|\mathrm{A}_{0}^{-1}(f)\right\|_{\mathrm{V}_{\beta, \delta}^{1}(\Omega)}}{\|f\|_{\mathrm{V}_{\beta, \delta}^{-1}(\Omega)}}+\sup _{f \in \mathrm{H}^{-1}\left(\Omega_{\delta}\right) \backslash\{0\}} \frac{\left\|\mathrm{A}_{\delta}^{-1}(f)\right\|_{\mathrm{V}_{\beta, \delta}^{1}\left(\Omega_{\delta}\right)}}{\|f\|_{\mathrm{V}_{\beta, \delta}^{-1}\left(\Omega_{\delta}\right)}}\right)<+\infty .
$$

Proof:

We need to provide an upper bound for both $\mathrm{A}_{\delta}^{-1}$ and $\mathrm{A}_{0}^{-1}$. We prove such a bound only for $\mathrm{A}_{\delta}^{-1}$, as the derivation for $\mathrm{A}_{0}^{-1}$ is very similar and slightly easier. First of all observe that, according to the uniform bounds (5), this is equivalent to proving that, for $\beta \in \mathbb{R}$ fixed such that $|\beta|<\beta_{\star}$, there exists a constant $C_{\beta}>0$ independent of $\delta$ such that

$$
C_{\beta} \leq \sup _{v \in \mathrm{H}_{0}^{1}\left(\Omega_{\delta}\right) \backslash\{0\}} \frac{\left|\left\langle\mathrm{A}_{\delta} \cdot \Upsilon_{\beta}^{\delta}(u), \Upsilon_{-\beta}^{\delta}(v)\right\rangle\right|}{\|u\|_{\mathrm{V}_{0, \delta}^{1}(\Omega)}\|v\|_{\mathrm{V}_{0, \delta}^{1}(\Omega)}} .
$$

We have $\left\langle\mathrm{A}_{\delta} \cdot \Upsilon_{\beta}^{\delta}(u), \Upsilon_{-\beta}^{\delta}(v)\right\rangle=\left(\nabla \Upsilon_{\beta}^{\delta}(u), \nabla \Upsilon_{-\beta}^{\delta}(v)\right)_{\Omega_{\delta}}$, according to the definition of $\mathrm{A}_{\delta}$. Expanding this expression yields

$$
\begin{aligned}
\left(\nabla \Upsilon_{+\beta}(u), \nabla \Upsilon_{-\beta}(v)\right)_{\Omega_{\delta}}=(\nabla u, \nabla v)_{\Omega_{\delta}} & \\
& +\beta \int_{\Omega} \nabla d_{\star}(\boldsymbol{x}) \cdot \frac{u \nabla v-v \nabla u}{d_{\delta}(\boldsymbol{x}) \ln d_{\delta}(\boldsymbol{x})} d \boldsymbol{x}-\beta^{2} \int_{\Omega} \frac{u v}{\left|d_{\delta}(\boldsymbol{x}) \ln d_{\delta}(\boldsymbol{x})\right|^{2}} d \boldsymbol{x} .
\end{aligned}
$$

Observe that, taking $v=\bar{u}$, the second term in the right hand side above is purely imaginary. In addition, since $\left|d_{\star}(\boldsymbol{x}) \ln d_{\star}(\boldsymbol{x})\right| \leq\left|d_{\delta}(\boldsymbol{x}) \ln d_{\delta}(\boldsymbol{x})\right|$ for all $\boldsymbol{x} \in \Omega$, using Proposition 4.1 below and the fact that $v \in \mathrm{H}_{0}^{1}\left(\Omega_{\delta}\right) \subset \mathrm{H}_{0}^{1}(\Omega)$, we obtain

$$
\begin{aligned}
\Re e\left\{\left(\nabla \Upsilon_{+\beta}(u), \nabla \Upsilon_{-\beta}(v)\right)_{\Omega_{\delta}}\right\} & \geq\left(1-\left|\beta / \beta_{\star}\right|^{2}\right)\|\nabla u\|_{\mathrm{L}^{2}\left(\Omega_{\delta}\right)}^{2} \\
& \geq \frac{1-\left|\beta / \beta_{\star}\right|^{2}}{1+1 / \beta_{\star}^{2}}\|u\|_{\mathrm{V}_{0, \delta}^{1}\left(\Omega_{\delta}\right)}^{2} .
\end{aligned}
$$


Inequality (8) leads to the conclusion of the proof since it yields the inf-sup condition (6) with a constant independent of $\delta$.

\section{Hardy type inequalities}

This section is dedicated to proving two inequalities that take a form similar to Hardy's inequality, but adapted to our geometrical setting. The first result below may be understood as a cylindrical version of Hardy's inequality. The proof, though, is made tricky by the rather general geometry under consideration here and, in particular, the possibility for $\Gamma$ to admit self-crossing points.

\section{Proposition 4.1.}

There exists a constant $\beta_{\star}>0$ such that

$$
\frac{1}{\beta_{\star}^{2}}:=\sup _{v \in \mathrm{H}_{0}^{1}(\Omega) \backslash\{0\}}\left\{\frac{1}{\|\nabla v\|_{\mathrm{L}^{2}(\Omega)}^{2}} \int_{\Omega} \frac{|v(\boldsymbol{x})|^{2} d \boldsymbol{x}}{\left|d_{\star}(\boldsymbol{x}) \ln d_{\star}(\boldsymbol{x})\right|^{2}}\right\}<+\infty .
$$

\section{Proof:}

During this proof, we shall refer to $e_{3}(t):=\partial_{t} \gamma(t) /\left|\partial_{t} \gamma(t)\right|$, consider a $\mathscr{C}^{0}$-vector field $t \mapsto \boldsymbol{e}_{1}(t) \in \mathbb{R}^{3}$ such that $\boldsymbol{e}_{1}(t) \cdot \boldsymbol{e}_{3}(t)=0, \forall t \in \mathbb{R}$, and set $\boldsymbol{e}_{2}(t):=\boldsymbol{e}_{3}(t) \times \boldsymbol{e}_{1}(t)$. Denoting $\mathrm{I}_{\epsilon}:=(-\epsilon,+\epsilon)$ and $\mathrm{D}_{\epsilon} \subset \mathbb{R}^{2}$ the disk of center 0 and radius $\epsilon$, for each $t \in \mathbb{R}$, there exists a small cylinder $\hat{Q}_{t}=\mathrm{D}_{\epsilon_{t}} \times \mathrm{I}_{\epsilon_{t}}$ with $\epsilon_{t}>0$ such that the map $\phi_{t}: \hat{Q}_{t} \rightarrow \mathbb{R}^{3}$ defined by

$$
\phi_{t}(x, y, z):=\gamma(t+z)+x \boldsymbol{e}_{1}(t)+y \boldsymbol{e}_{2}(t)
$$

is an immersion. Pick finitely many $t_{1}, t_{2}, \ldots t_{n}$ such that $[0, L] \subset \cup_{j=1}^{n}\left(t_{j}-\epsilon_{t_{j}}, t_{j}+\epsilon_{t_{j}}\right)$. Denote $\hat{Q}_{j}:=\hat{Q}_{t_{j}}, Q_{j}:=\phi_{t_{j}}\left(\hat{Q}_{j}\right)$ and $\Gamma_{j}:=\Gamma \cap Q_{j}$ so that, in particular, $\Gamma \subset Q_{1} \cup \cdots \cup Q_{n}$.

Let $\omega_{\eta}=\{\boldsymbol{x} \in \Omega \mid d(\boldsymbol{x})<\eta\}$. Let us prove that, if $\eta$ is chosen small enough, then for any $\boldsymbol{x} \in \omega_{\eta}$ there is one $j$ such that $\boldsymbol{x} \in Q_{j}$ and $d(\boldsymbol{x})=\inf _{\boldsymbol{y} \in \Gamma_{j}}|\boldsymbol{x}-\boldsymbol{y}|$. To show this, proceed by contradiction, assuming for a moment that such is not the case.

This means that there exists a sequence $\boldsymbol{x}_{p} \in \Omega$ with $\lim _{p \rightarrow \infty} d\left(\boldsymbol{x}_{p}\right)=0$ and such that, for any $\boldsymbol{x}_{p}^{*} \in \Gamma$ satisfying $\left|\boldsymbol{x}_{p}-\boldsymbol{x}_{p}^{*}\right|=d\left(\boldsymbol{x}_{p}\right)$, none of the $Q_{j}$ 's both contain $\boldsymbol{x}_{p}$ and $\boldsymbol{x}_{p}^{*}$. Since $\Gamma$ is compact, extracting a sub-sequence if necessary, we may assume that $\lim _{p \rightarrow \infty} \boldsymbol{x}_{p}=\lim _{p \rightarrow \infty} \boldsymbol{x}_{p}^{*}=\boldsymbol{x}_{\infty} \in \Gamma$. Take a $Q_{m}$ containing $\boldsymbol{x}_{\infty}$. Then, since $Q_{m}$ is an open neighborhood of $\boldsymbol{x}_{\infty}$, for sufficiently large $p$ we have both $\boldsymbol{x}_{p} \in Q_{m}$ and $\boldsymbol{x}_{p}^{*} \in Q_{m}$ which contradicts our initial assumption.

From now on, we assume that $\eta>0$ is chosen small enough to guarantee the property discussed in the previous paragraph. Denote $d_{j}(\boldsymbol{x})=\inf _{\boldsymbol{y} \in \Gamma_{j}}|\boldsymbol{x}-\boldsymbol{y}|$, and let $\mathbf{1}_{Q_{j}}$ refer to the characteristic function of $Q_{j}$. What precedes shows that, for any $\boldsymbol{x} \in \omega_{\eta}$, we have

$$
\frac{1}{\left|d_{\star}(\boldsymbol{x}) \ln d_{\star}(\boldsymbol{x})\right|} \leq \sum_{j=1}^{n} \frac{\mathbf{1}_{Q_{j}}(\boldsymbol{x})}{\left|d_{j}(\boldsymbol{x}) \ln d_{j}(\boldsymbol{x})\right|}
$$

Choose a smooth open set $Q_{0} \subset \mathbb{R}^{3}$ such that $Q_{0} \cup Q_{1} \cdots \cup Q_{n}$ achieves a covering of $\bar{\Omega}$. Using a partition of unity subordinated to this covering, one may decompose any $v \in \mathrm{H}_{0}^{1}(\Omega)$ in the form $v=v_{0}+\cdots+v_{n}$ where $v_{j} \in \mathrm{H}_{0}^{1}\left(Q_{j}\right)$. This remark, together with Inequality (10), shows that it suffices to prove the existence of a constant $C>0$ such that

$$
\int_{Q_{j}} \frac{|v(\boldsymbol{x})|^{2} d \boldsymbol{x}}{\left|d_{j}(\boldsymbol{x}) \ln d_{j}(\boldsymbol{x})\right|^{2}} \leq C \int_{Q_{j}}|\nabla v|^{2} d \boldsymbol{x} \quad \forall v \in \mathrm{H}_{0}^{1}\left(Q_{j}\right) \quad \forall j=1 \ldots n .
$$


From now on, and until the end of the proof, we take a $j$ fixed. Let us relabel for a moment $\epsilon=\epsilon_{t_{j}}, \phi=\phi_{t_{j}}$ and set $\hat{Q}=\hat{Q}_{j}=\mathrm{D}_{\epsilon} \times \mathrm{I}_{\epsilon}, \Sigma:=\{0\} \times \mathrm{I}_{\epsilon}$ and $\hat{v}=v \circ \phi$. For any $\hat{\boldsymbol{x}} \in \hat{Q}$ we also set $\hat{d}(\hat{\boldsymbol{x}})=\inf _{\hat{\boldsymbol{y}} \in \Sigma}|\hat{\boldsymbol{x}}-\hat{\boldsymbol{y}}|$. The diffeomorphism $\phi$ and its inverse $\phi^{-1}$ are both Lipschitz, so there are constants $c_{ \pm}>0$ such that $c_{-}|\hat{\boldsymbol{x}}-\hat{\boldsymbol{y}}| \leq|\phi(\hat{\boldsymbol{x}})-\phi(\hat{\boldsymbol{y}})| \leq c_{+}|\hat{\boldsymbol{x}}-\hat{\boldsymbol{y}}|$. Routine verifications show that this implies existence of two constants $c^{\prime}, c^{\prime \prime}>0$ such that

$$
c^{\prime} \hat{d}(\hat{\boldsymbol{x}}) \leq d(\phi(\hat{\boldsymbol{x}})) \leq c^{\prime \prime} \hat{d}(\hat{\boldsymbol{x}}) \quad \forall \hat{\boldsymbol{x}} \in \hat{Q} .
$$

Let $r, \theta$ refer to the polar coordinates in $\mathbb{R}^{2}$ so that the cylinder $\hat{Q}$ is parametrized by the cylindrical coordinates $(r, \theta, z) \in[0,+\epsilon) \times[0,2 \pi] \times \mathrm{I}_{\epsilon}$. The classical Hardy's inequality applied in $\hat{Q}$, see [8], shows that

$$
\int_{\hat{Q}} \frac{|\hat{v}|^{2} r d r d \theta d z}{|r \ln (r)|^{2}} \leq C \int_{\hat{Q}}|\nabla \hat{v}|+|\hat{v}|^{2} d \hat{\boldsymbol{x}} \quad \forall \hat{v} \in \mathrm{H}^{1}(\hat{Q}) .
$$

There only remains to observe that $\hat{d}(\hat{\boldsymbol{x}})=r$, to use (12), and to apply the change of variables $\boldsymbol{x}=\phi(\hat{\boldsymbol{x}})$ in the integrals (13). Denoting $D \phi$ the differential of $\phi$, since $\|D \phi\|_{L^{\infty}(\hat{Q})}$ and $\left\|D \phi^{-1}\right\|_{L^{\infty}\left(Q_{j}\right)}$ are both bounded, this finally yields inequality (11) with $\|\nabla v\|_{\mathrm{L}^{2}\left(Q_{j}\right)}^{2}+\|v\|_{\mathrm{L}^{2}\left(Q_{j}\right)}^{2}$ instead of just $\|\nabla v\|_{\mathrm{L}^{2}(\Omega)}^{2}$. We conclude by using Poincare's inequality in $Q_{j}$.

We will also need another $\delta$-dependent weighted inequality. This one involves a weighted $\mathrm{L}^{2}$-norm evaluated only over a coronal cylinder of radius $\delta$, and is not primarily based on the classical Hardy's inequality. Instead it is derived by means of Kondratiev's analysis.

Proposition 4.2.

Denoting $\mathbb{Q}_{\delta}:=\{\boldsymbol{x} \in \Omega \mid \delta<d(\boldsymbol{x})<2 \delta\}$ we have

$$
\limsup _{\delta \rightarrow 0} \sup _{v \in \mathrm{H}^{2}(\Omega) \cap \mathrm{H}_{0}^{1}(\Omega) \backslash\{0\}}\left\{\frac{1}{\|\Delta v\|_{\mathrm{L}^{2}(\Omega)}^{2}} \int_{\mathbb{Q}_{\delta}}\left|\frac{v(\boldsymbol{x})}{d_{\star}(\boldsymbol{x})}\right|^{2} d \boldsymbol{x}\right\}<+\infty .
$$

Proof:

To establish this result, we follow a path similar to that of the proof of Proposition 4.1, and use the same notations. Take any $\boldsymbol{x} \in \omega_{\eta}$. According to the first part of the preceding proof, there exists $j$ such that $\boldsymbol{x} \in Q_{j}$ and $d_{j}(\boldsymbol{x})=d_{\star}(\boldsymbol{x})$, hence $\delta<d(\boldsymbol{x})<2 \delta \Rightarrow \delta<$ $d_{j}(\boldsymbol{x})<2 \delta$. As a consequence $\mathbb{Q}_{\delta} \subset \cup_{j=1}^{n} \mathbb{Q}_{\delta}^{j}$ where $\mathbb{Q}_{\delta}^{j}:=\left\{\boldsymbol{x} \in Q_{j}, \delta<d_{j}(\boldsymbol{x})<2 \delta\right\}$ which implies $\mathbf{1}_{\mathbb{Q}_{\delta}} \leq \sum_{j=1}^{n} \mathbf{1}_{\mathbb{Q}_{\delta}^{j}}$. Hence it suffices to prove, for each $j$, the existence of constants $C, \delta_{0}>0$ independent of $\delta$ such that

$$
\int_{\mathbb{Q}_{\delta}^{j}}\left|\frac{v(\boldsymbol{x})}{d_{j}(\boldsymbol{x})}\right|^{2} d \boldsymbol{x} \leq C \int_{\Omega}|\Delta v|^{2} d \boldsymbol{x} \quad \forall v \in \mathrm{H}^{2}(\Omega) \cap \mathrm{H}_{0}^{1}(\Omega), \quad \forall \delta \in\left(0, \delta_{0}\right) .
$$

Fixing $j$, and using a change of variables like in the previous proof, the inequality above boils down to establishing the existence of constants $C, \delta_{0}>0$ independent of $\delta$ such that, for any $v \in \mathrm{H}^{2}(\hat{Q}) \cap \mathrm{H}_{0}^{1}(\hat{Q})$ and any $\delta \in\left(0, \delta_{0}\right)$ we have

$$
\int_{\hat{Q}_{\delta}} \frac{|v|^{2}}{r^{2}} r d r d \theta d z \leq C \int_{\hat{Q}}|\Delta v|^{2}+|\nabla v|^{2}+|v|^{2} d \hat{\boldsymbol{x}} .
$$

where we recall that $\hat{Q}=\mathrm{D}_{\epsilon} \times \mathrm{I}_{\epsilon}$, the variables $r, \theta, z$ refer to the cylindrical coordinates in $\hat{Q}$, and $\hat{Q}_{\delta}:=\{\hat{\boldsymbol{x}} \in \hat{Q} \mid \delta<r<2 \delta\}$. To establish (14), set $\Delta_{\perp} v=r^{-2}\left(\left(r \partial_{r}\right)^{2}+\partial_{\theta}^{2}\right) v$. Decompose each point $\hat{\boldsymbol{x}} \in \hat{Q}$ as $\hat{\boldsymbol{x}}=\left(\hat{\boldsymbol{x}}_{\perp}, z\right)$, so that $\hat{\boldsymbol{x}}_{\perp}=(r \cos \theta, r \sin \theta)$ and $r=\left|\hat{\boldsymbol{x}}_{\perp}\right|$. Introduce the Fourier decomposition of $v$ in the $z$ variable, setting

$$
v_{p}\left(\hat{\boldsymbol{x}}_{\perp}\right):=\frac{1}{2 \epsilon} \int_{-\epsilon}^{+\epsilon} v\left(\hat{\boldsymbol{x}}_{\perp}, z\right) \exp (-i \pi z / \epsilon) d z .
$$


Then we have $v_{p} \in \mathrm{H}^{2}\left(\mathrm{D}_{\epsilon}\right) \cap \mathrm{H}_{0}^{1}\left(\mathrm{D}_{\epsilon}\right)$ and $-\Delta_{\perp} v_{p} \in \mathrm{L}^{2}\left(\mathrm{D}_{\epsilon}\right)$ for all $p \in \mathbb{Z}$, if $v \in \mathrm{H}^{2}(\hat{Q}) \cap$ $\mathrm{H}_{0}^{1}(\hat{Q})$. Applying Kondratiev's analysis in $\mathrm{D}_{\epsilon} \backslash\{0\}$, see Chapter 6 of [13], we find the existence of coefficients $\alpha_{p} \in \mathbb{C}$ and a constant $C>0$ independent of $p$ such that

$$
\left|\alpha_{p}\right|^{2}+\int_{\mathrm{D}_{\epsilon}} \frac{\left|v_{p}\left(\hat{\boldsymbol{x}}_{\perp}\right)-\alpha_{p}\right|^{2}}{\left|\hat{\boldsymbol{x}}_{\perp}\right|^{3}} d \hat{\boldsymbol{x}}_{\perp} \leq C \int_{\mathrm{D}_{\epsilon}}\left|\Delta_{\perp} v_{p}\right|^{2} d \hat{\boldsymbol{x}}_{\perp} \quad \forall p \in \mathbb{Z} .
$$

Parseval identity associated to Decomposition (15), together with the estimate above, indicates that $\sum_{p=-\infty}^{+\infty}\left|\alpha_{p}\right|^{2}<+\infty$ so that there exists a function $\alpha=\alpha(z) \in \mathrm{L}^{2}\left(\mathrm{I}_{\epsilon}\right)$ and constants $C, C^{\prime}>0$ independent of $v$ satisfying

$$
\begin{aligned}
& \|\alpha\|_{\mathrm{L}^{2}\left(\mathrm{I}_{\epsilon}\right)}^{2}+\int_{\hat{Q}} \frac{\left|v\left(\hat{\boldsymbol{x}}_{\perp}, z\right)-\alpha(z)\right|^{2}}{\left|\hat{\boldsymbol{x}}_{\perp}\right|^{3}} d \hat{\boldsymbol{x}}_{\perp} d z \\
& \quad \leq C\|f\|_{\mathrm{L}^{2}(\hat{Q})}^{2}=C\left\|\Delta_{\perp} v\right\|_{\mathrm{L}^{2}(\hat{Q})}^{2} \leq C^{\prime}\|\Delta v\|_{\mathrm{L}^{2}(\hat{Q})}^{2} \quad \forall v \in \mathrm{H}^{2}(\hat{Q}) \cap \mathrm{H}_{0}^{1}(\hat{Q}) .
\end{aligned}
$$

The inequality above is justified by standard elliptic a priori estimates for the Laplace operator, see [13, Chap.3] for example. Finally, let us pick an arbitrary $v \in \mathrm{H}^{2}(\hat{Q}) \cap \mathrm{H}_{0}^{1}(\hat{Q})$. Plugging (16) into the left hand side of (14) yields

$$
\begin{gathered}
\int_{\hat{Q}_{\delta}} \frac{|v|^{2}}{r^{2}} r d r d \theta d z=\int_{\hat{Q}_{\delta}} \frac{|v(\hat{\boldsymbol{x}})|^{2}}{\left|\hat{\boldsymbol{x}}_{\perp}\right|^{2}} d \hat{\boldsymbol{x}} \leq \int_{\hat{Q}_{\delta}} \frac{|\alpha(z)|^{2}}{\left|\hat{\boldsymbol{x}}_{\perp}\right|^{2}} d \hat{\boldsymbol{x}}+\int_{\hat{Q}} \frac{|v(\hat{\boldsymbol{x}})-\alpha(z)|^{2}}{\left|\hat{\boldsymbol{x}}_{\perp}\right|^{3}} d \hat{\boldsymbol{x}} \\
\leq\|\alpha\|_{\mathrm{L}^{2}\left(\mathrm{I}_{\epsilon}\right)}^{2} \underbrace{\left(\int_{\delta}^{2 \delta} \frac{d r}{r}\right)}_{=\ln (2)}+C\|\Delta v\|_{\mathrm{L}^{2}(\hat{Q})}^{2} \leq C^{\prime}\|\Delta v\|_{\mathrm{L}^{2}(\hat{Q})}^{2} .
\end{gathered}
$$

Since $v$ was chosen arbitrarily in $\mathrm{H}^{2}(\hat{Q}) \cap \mathrm{H}_{0}^{1}(\hat{Q})$, and the constant $C^{\prime}>0$ is independent of $\delta$, this concludes the proof.

\section{Norm convergence of the resolvent}

We will now use the previous analysis to show that $\mathrm{A}_{\delta}^{-1}$ strongly converges toward $\mathrm{A}_{0}^{-1}$ in some appropriate operator norm. Before stating this result let us just point out that, using extension by 0 , we have $\mathrm{H}_{0}^{1}\left(\Omega_{\delta}\right) \subset \mathrm{H}_{0}^{1}(\Omega)$ so that $\mathrm{H}^{-1}(\Omega) \subset \mathrm{H}^{-1}\left(\Omega_{\delta}\right)$. The expression $\mathrm{A}_{\delta}^{-1}(f)$ with $f \in \mathrm{H}^{-1}(\Omega)$ should be understood according to these inclusions.

\section{Proposition 5.1.}

For $\beta_{\star}>0$ as in Proposition 3.1, and for each $\beta \in \mathbb{R}$ satisfying $|\beta|<\beta_{\star}$, there exist constants $c_{\beta}, \delta_{0}>0$ independent of $\delta$, such that

$$
\sup _{f \in \mathrm{L}^{2}(\Omega) \backslash\{0\}} \frac{\left\|\mathrm{A}_{0}^{-1}(f)-\mathrm{A}_{\delta}^{-1}(f)\right\|_{\mathrm{V}_{\beta, \delta}^{1}(\Omega)}}{\|f\|_{\mathrm{L}^{2}(\Omega)}} \leq \frac{c_{\beta}}{|\ln \delta|^{\beta}} \quad \forall \delta \in\left(0, \delta_{0}\right) .
$$

\section{Proof:}

First of all consider a $\mathscr{C}^{\infty}$ cut-off function $\chi: \mathbb{R} \rightarrow \mathbb{R}$ such that $\chi(t)=0$ for $t \leq 1$ and $\chi(t)=1$ for $t \geq 2$, and set $\chi_{\delta}(\boldsymbol{x}):=\chi(d(\boldsymbol{x}) / \delta)$ and $\psi_{\delta}:=1-\chi_{\delta}$. In the remaining of this proof, we shall denote $\mathbb{Q}_{\delta}:=\operatorname{supp}\left(\nabla \chi_{\delta}\right)$ so that, for any $\boldsymbol{x} \in \mathbb{Q}_{\delta}$, we have $\delta \leq d(\boldsymbol{x}) \leq 2 \delta$. We introduce the operator $\mathrm{R}_{\delta}: \mathrm{H}^{-1}(\Omega) \rightarrow \mathrm{H}_{0}^{1}\left(\Omega_{\delta}\right)$ defined by

$$
\mathrm{R}_{\delta}(f):=\chi_{\delta} \mathrm{A}_{0}^{-1}(f) \quad \forall f \in \mathrm{H}^{-1}(\Omega) .
$$

Now take any $f \in \mathrm{L}^{2}(\Omega)$, and set $u_{\delta}:=\mathrm{A}_{\delta}^{-1}(f) \in \mathrm{H}_{0}^{1}\left(\Omega_{\delta}\right), \hat{u}_{\delta}:=\mathrm{R}_{\delta}(f) \in \mathrm{H}_{0}^{1}\left(\Omega_{\delta}\right)$ and $u_{0}:=\mathrm{A}_{0}^{-1}(f) \in \mathrm{H}^{2}(\Omega) \cap \mathrm{H}_{0}^{1}(\Omega)$. Let us look at the problem solved by the difference 
$u_{\delta}-\hat{u}_{\delta}$. There exist constants $c, c^{\prime}>0$ independent of $\delta$ such that, for any $v \in \mathrm{H}_{0}^{1}\left(\Omega_{\delta}\right)$, we have

$$
\begin{aligned}
\left|\left\langle\mathrm{A}_{\delta}\left(u_{\delta}-\hat{u}_{\delta}\right), v\right\rangle\right| & =\left|\left(\nabla\left(u_{\delta}-\hat{u}_{\delta}\right), \nabla v\right)_{\Omega}\right| \\
& =\left|\left(f, \psi_{\delta} v\right)_{\Omega}+\left(\nabla \chi_{\delta}, u_{0} \nabla v-v \nabla u_{0}\right)_{\mathbb{Q}_{\delta}}\right| \\
& \leq\|f\|_{L^{2}(\Omega)}\left\|\psi_{\delta} v\right\|_{\mathrm{L}^{2}(\Omega)}+\left|\left(\nabla \chi_{\delta}, u_{0} \nabla v-v \nabla u_{0}\right)_{\mathbb{Q}_{\delta}}\right| .
\end{aligned}
$$

To derive an upper bound for the second term in the right hand side above, observe that $\delta \leq d(\boldsymbol{x}) \leq 2 \delta$ for $\boldsymbol{x} \in \operatorname{supp}\left(\nabla \chi_{\delta}\right)$. Since $|\nabla d| \leq 1$, we have $\sup _{\boldsymbol{x} \in \bar{\Omega}}\left|\nabla \chi_{\delta}\right| \leq$ $2 d(\boldsymbol{x})^{-1} \sup _{\boldsymbol{x} \in \bar{\Omega}}\left|\partial_{t} \chi\right|$. So Cauchy-Schwarz inequality, together with Proposition 4.1 applied to $\nabla u_{0}$, and Proposition 4.2 applied to $u_{0}$, yield the existence of a constant $C>0$ independent of $\delta$ such that

$$
\begin{aligned}
\left|\left(\nabla \chi_{\delta}, u_{0} \nabla v-v \nabla u_{0}\right)_{\mathbb{Q}_{\delta}}\right| & \leq C\left(\|\nabla v\|_{\mathrm{L}^{2}\left(\mathbb{Q}_{\delta}\right)}+\|v\|_{\mathrm{L}^{2}\left(\mathbb{Q}_{\delta}\right)}\right)\left\|\Delta u_{0}\right\|_{\mathrm{L}^{2}(\Omega)} \\
& \leq C^{\prime}|\ln \delta|^{-\beta}\|v\|_{\mathrm{V}_{-\beta, \delta}^{1}(\Omega)}\|f\|_{\mathrm{L}^{2}(\Omega)} .
\end{aligned}
$$

The first term in the right hand side of (17) can be bounded by noting that $\delta \leq d_{\delta}(\boldsymbol{x}) \leq 2 \delta$ on $\operatorname{supp}\left(\psi_{\delta}\right)$. So applying Hardy's inequality (9) yields a constant $C>0$ independent of $\delta$ such that $\left\|\psi_{\delta} v\right\|_{\mathrm{L}^{2}(\Omega)} \leq C \delta|\ln \delta|^{1-\beta}\|v\|_{\mathrm{V}_{-\beta, \delta}^{1}(\Omega)}$ for all $v \in \mathrm{H}_{0}^{1}\left(\Omega_{\delta}\right)$. Plugging this together with Estimate (18) into Inequality (17) provides a constant $C>0$ independent of $\delta$ such that

$$
\left|\left\langle\mathrm{A}_{\delta}\left(u_{\delta}-\hat{u}_{\delta}\right), v\right\rangle\right| \leq C|\ln \delta|^{-\beta}\|v\|_{\mathrm{V}_{-\beta, \delta}^{1}(\Omega)}\|f\|_{\mathrm{L}^{2}(\Omega)} .
$$

Observe that $\mathrm{A}_{\delta}\left(u_{\delta}-\hat{u}_{\delta}\right)=\left(\mathrm{Id}-\mathrm{A}_{\delta} \mathrm{R}_{\delta}\right) f \in \mathrm{H}^{-1}(\Omega)$. Since $v \in \mathrm{H}_{0}^{1}\left(\Omega_{\delta}\right)$ was arbitrary in the calculus above, what precedes shows that there exists a constant $C>0$ independent of $\delta$ such that

$$
\left\|\left(\mathrm{Id}-\mathrm{A}_{\delta} \mathrm{R}_{\delta}\right) f\right\|_{\mathrm{V}_{\beta, \delta}^{-1}\left(\Omega_{\delta}\right)} \leq C\|f\|_{\mathrm{L}^{2}(\Omega)}|\ln \delta|^{-\beta} .
$$

Following the same calculus, but choosing a test function $v \in \mathrm{H}_{0}^{1}(\Omega)$, the same result as (19) holds with $\mathrm{A}_{\delta}, \Omega_{\delta}$ replaced by $\mathrm{A}_{0}, \Omega$. To conclude the proof, there only remains to use the stability estimates of Proposition 3.1 that yield constants $C>0$ independent of $\delta$ such that, for any $f \in \mathrm{L}^{2}(\Omega)$, we have

$$
\begin{aligned}
\left\|\mathrm{A}_{0}^{-1}(f)-\mathrm{A}_{\delta}^{-1}(f)\right\|_{\mathrm{V}_{\beta, \delta}^{1}(\Omega)} & \leq\left\|\mathrm{A}_{0}^{-1}(f)-\mathrm{R}_{\delta}(f)\right\|_{\mathrm{V}_{\beta, \delta}^{1}(\Omega)}+\left\|\mathrm{A}_{\delta}^{-1}(f)-\mathrm{R}_{\delta}(f)\right\|_{\mathrm{V}_{\beta, \delta}^{1}\left(\Omega_{\delta}\right)} \\
& \leq C\left(\left\|f-\mathrm{A}_{0} \mathrm{R}_{\delta}(f)\right\|_{\mathrm{V}_{\beta, \delta}^{-1}(\Omega)}+\left\|f-\mathrm{A}_{\delta} \mathrm{R}_{\delta}(f)\right\|_{\mathrm{V}_{\beta, \delta}^{-1}\left(\Omega_{\delta}\right)}\right) \\
& \leq C|\ln \delta|^{-\beta}\|f\|_{\mathrm{L}^{2}(\Omega)} .
\end{aligned}
$$

The proposition above yields consistency estimates for the asymptotic source problem. Indeed, let $f \in \mathrm{L}^{2}(\Omega)$ refer to a fixed function not depending on $\delta$, let $u_{0} \in \mathrm{H}_{0}^{1}\left(\Omega_{\delta}\right)$ satisfy $-\Delta u_{0}=f$ in $\Omega$, and let $u_{\delta} \in \mathrm{H}_{0}^{1}\left(\Omega_{\delta}\right)$ satisfy $-\Delta u_{\delta}=f$ in $\Omega_{\delta}$. Then Proposition 5.1 implies that $\left\|u_{\delta}-u_{0}\right\|_{\mathrm{H}^{1}(\Omega \backslash \bar{U})}=O\left(|\ln \delta|^{-\beta}\right)$ for any neighborhood $U$ of $\Gamma$, and for $|\beta|<\beta_{\star}$.

Sharper results can be obtained in terms of the $\mathrm{L}^{2}$-norm. Observe that there exists a constant $C>0$ independent of $\delta$ such that $\|v\|_{\mathrm{L}^{2}(\Omega)} \leq C\|v\|_{\mathrm{V}_{\beta, \delta}^{1}(\Omega)}$ for any $\beta \in \mathbb{R}$. Plugging this into the estimate of Proposition 5.1 yields the following result.

Corollary 5.1.

For $\beta_{\star}>0$ as in Proposition 3.1, and for any $\epsilon \in\left(0,2 \beta_{\star}\right)$, there exist constants $c_{\epsilon}, \delta_{0}>0$ 
independent of $\delta$ such that

$$
\sup _{f \in \mathrm{L}^{2}(\Omega) \backslash\{0\}} \frac{\left\|\mathrm{A}_{0}^{-1}(f)-\mathrm{A}_{\delta}^{-1}(f)\right\|_{\mathrm{L}^{2}(\Omega)}}{\|f\|_{\mathrm{L}^{2}(\Omega)}} \leq \frac{c_{\epsilon}}{|\ln \delta|^{\beta_{\star}-\epsilon}} \quad \forall \delta \in\left(0, \delta_{0}\right) .
$$

Recall that $\mathrm{L}^{2}(\Omega) \subset \mathrm{H}^{-1}\left(\Omega_{\delta}\right)$, and $\mathrm{H}_{0}^{1}\left(\Omega_{\delta}\right) \subset \mathrm{L}^{2}(\Omega)$, so that $\mathrm{A}_{\delta}^{-1}$ is a continuous operator mapping $\mathrm{L}^{2}(\Omega)$ to $\mathrm{L}^{2}(\Omega)$. As such, it is self-adjoint and compact and, as can be checked by routine verifications, its eigenvalues with those of $\mathrm{A}_{\delta}^{-1}$ considered as an operator mapping $\mathrm{L}^{2}\left(\Omega_{\delta}\right)$ to $\mathrm{L}^{2}\left(\Omega_{\delta}\right)$. Then, since $\mathrm{A}_{0}^{-1}: \mathrm{L}^{2}(\Omega) \rightarrow \mathrm{L}^{2}(\Omega)$ is also self-adjoint and compact, straightforward application of Theorem 4.10 of Chapter $\mathrm{V}$ of [12] yields that the spectra of $\mathrm{A}_{\delta}^{-1}$ and $\mathrm{A}_{0}^{-1}$ are closed to each other.

\section{Proposition 5.2.}

For $\beta_{\star}>0$ as in Proposition 3.1, and for any $\epsilon \in\left(0,2 \beta_{\star}\right)$, there exist constants $\delta_{0}, c_{\epsilon}>0$ independent of $\delta$ such that

$$
\sup _{\mu \in \mathfrak{S}\left(\mathrm{A}_{0}\right)} \inf _{\lambda \in \mathfrak{S}\left(\mathrm{A}_{\delta}\right)}\left|\frac{1}{\mu}-\frac{1}{\lambda}\right|+\sup _{\mu \in \mathfrak{S}\left(\mathrm{A}_{\delta}\right)} \inf _{\lambda \in \mathfrak{S}\left(\mathrm{A}_{0}\right)}\left|\frac{1}{\mu}-\frac{1}{\lambda}\right| \leq \frac{c_{\epsilon}}{|\ln \delta|^{\beta_{\star}-\epsilon}} \quad \forall \delta \in\left(0, \delta_{0}\right) .
$$

\section{References}

[1] H. Ammari. An introduction to mathematics of emerging biomedical imaging, volume 62 of Mathématiques $\&$ Applications (Berlin) [Mathematics $\&$ Applications]. Springer, Berlin, 2008.

[2] H. Ammari and H. Kang. Reconstruction of small inhomogeneities from boundary measurements, volume 1846 of Lecture Notes in Mathematics. Springer-Verlag, Berlin, 2004.

[3] I.I. Argatov and F.J. Sabina. Acoustic diffraction by a thin soft torus. Wave Motion, $45(6): 846-856,2008$.

[4] A.L. Dontchev and R.T. Rockafellar. Implicit functions and solution mappings. Springer Series in Operations Research and Financial Engineering. Springer, New York, second edition, 2014. A view from variational analysis.

[5] M.V. Fedoryuk. Asymptotics of the solution of the Dirichlet problem for the Laplace and helmholtz equations in the exterior of a slender cylinder. Izv. Akad. Nauk SSSR Ser. Mat., 45(1):167-186, 1981.

[6] M.V. Fedoryuk. Theory of Cubature Formulas and the Applications of Functionnal Analysis to Problems of Mathematical Physics, volume 126 of AMS Trans., chapter The Dirichlet problem for the Laplace operator in the exterior of a thin body of revolution. American Mathematical Society, 1985.

[7] J. Geer. The scattering of a scalar wave by a slender body of revolution. SIAM J. Appl. Math., 34(2):348-370, 1978.

[8] G. H. Hardy, J. E. Littlewood, and G. Pólya. Inequalities. Cambridge Mathematical Library. Cambridge University Press, Cambridge, 1988. Reprint of the 1952 edition.

[9] A. M. Il'in. Matching of asymptotic expansions of solutions of boundary value problems, volume 102 of Translations of Mathematical Monographs. American Mathematical Society, Providence, RI, 1992. Translated from the Russian by V. Minachin [V. V. Minakhin].

[10] A.M. Il'in. Study of the asymptotic behavior of the solution of an elliptic boundary value problem in a domain with a small hole. Trudy Sem. Petrovsk., (6):57-82, 1981. 
[11] D.S. Jones. Methods in electromagnetic wave propagation. The Clarendon Press, Oxford University Press, New York, 1979. Oxford Engineering Science Series.

[12] T. Kato. Perturbation theory for linear operators. Classics in Mathematics. SpringerVerlag, Berlin, 1995. Reprint of the 1980 edition.

[13] V. A. Kozlov, V. G. Maz'ya, and J. Rossmann. Elliptic Boundary Value Problems in Domains with Point Singularities, volume 52 of Mathematical Surveys and Monographs. AMS, Providence, 1997.

[14] V. Maz'ya, S. Nazarov, and B. Plamenevskij. Asymptotic theory of elliptic boundary value problems in singularly perturbed domains. Vol. I \& II, volume 111 \& 112 of Operator Theory: Advances and Applications. Birkhäuser Verlag, Basel, 2000.

[15] V. G. Maz'ya, S. A. Nazarov, and B. A. Plamenevskii. The asymptotic behavior of solutions of the Dirichlet problem in a domain with a cut out thin tube. Mat. Sb. (N.S.), 116(158)(2):187-217, 1981.

[16] V.G. Maz'ya, S.A. Nazarov, and B.A. Plamenevskiu. Asymptotic expansions of eigenvalues of boundary value problems for the Laplace operator in domains with small openings. Izv. Akad. Nauk SSSR Ser. Mat., 48(2):347-371, 1984.

[17] S.A. Nazarov. Asymptotic conditions at a point, selfadjoint extensions of operators, and the method of matched asymptotic expansions. In Proceedings of the St. Petersburg Mathematical Society, Vol. V, volume 193 of Amer. Math. Soc., Transl. Ser. 2, pages 77-125, Providence, RI, 1999.

[18] S.A. Nazarov and J. Sokołowski. Asymptotic analysis of shape functionals. J. Math. Pures Appl., 82(2):125-196, 2003.

[19] S.A. Nazarov and J. Sokołowski. Self-adjoint extensions for the Neumann Laplacian and applications. Acta Math. Sin. (Engl. Ser.), 22(3):879-906, 2006.

[20] A.A. Novotny and J. Sokołowski. Topological derivatives in shape optimization. Interaction of Mechanics and Mathematics. Springer, Heidelberg, 2013.

[21] M. Yu. Planida. On the convergence of solutions of singularly perturbed boundary value problems for the Laplacian. Mat. Zametki, 71(6):867-877, 2002.

[22] G. V. Zhdanova. The Dirichlet problem for the Helmholtz operator in the exterior of a thin body of revolution. Differentsial' nye Uravneniya, 20(8):1403-1411, 1984. 